\title{
Wolfgang Horn
}

\section{Andreas Raselius Ambergensis als Verehrer Glareans - Eine Miszelle zur Glarean-Rezeption um 1600}

Andreas Raselius war als Angehöriger des Kollegiums am Regensburger Gymnasium poeticum über 15 Jahre lang für Latein- und Musikunterricht zuständig. Er war ein humanistisch gebildeter Mann, der in und neben seinen Berufspflichten unablässig komponierte, fremde Musikstücke abschrieb und auch Bücher über verschiedene Gegenstände verfasste. Amberg in der Oberpfalz als Ort der Herkunft, Regensburg als die wichtigste Wirkungs- und Überlieferungsstätte und schließlich Heidelberg als letzter Tätigkeits- und auch Sterbeort umreißen den regionalen Rahmen seines Wirkens.

Andreas Raselius wurde um 1563 als Sohn des evangelisch-lutherischen Pfarrers der Gemeinde Hahnbach bei Amberg in der Oberpfalz geboren. Nach Lateinschuljahren in Amberg, wo er durch den dortigen Organisten Matthias Gastritz u. a. die Motettenkunst des Orlando di Lasso kennen lernte, und dem nahen Sulzbach bezog Raselius - wie das für begabte Oberpfälzer aufgrund der politischen Verhältnisse fast zwingend war - die Universität Heidelberg. Von Ende 1581 bis ins Jahr 1584 studierte er an der dortigen Artistenfakultät, die er mit dem Magistergrad verließ. Sein Weg führte ihn in die lutherische freie Reichsstadt Regensburg. ${ }^{1}$ Am dortigen Gymnasium poeticum wurde er im April 1584 Lehrer der zweiten Klasse. Eine nur flüchtige Lektüre von Schulordnungen des 16. und 17. Jahrhunderts zeigt, wie stark der Gymnasialunterricht dieser Zeit auf das Ideal einer lateinischen Eloquenz hin orientiert war; 2 die viel zitierte Formel des Straßburger Schulmannes Johannes Sturm fasste das protestantische Bildungsideal in die einprägsamen Worte einer »sapiens atque eloquens pietas ${ }^{3}{ }^{3}$ Raselius war

1 Die Stadt Regensburg bekannte sich im Jahre 1542 zur lutherischen Konfession und bewahrte diese bis zum Ende des Alten Reiches (1803/06), also über 260 Jahre lang. Die bei der Reformation bestehenden katholischen Klöster und Stifte blieben aber unberührt - ein beherzigenswertes Beispiel konfessioneller Toleranz.

2 Das große Werk von Reinhold Vormbaum, Die evangelischen Schulordnungen des sechszehnten [siebenzehnten, achtzehnten] Jahrhunderts, 3 Bde., Gütersloh 1860, 1863 und 1864, enthält zwar keine Regensburger Schulordnung aus der Zeit des Raselius, liefert aber vielfältiges Anschauungsmaterial. Entsprechendes gilt (aufgrund der Quellenlage) für das Buch von Klaus Wolfgang Niemöller, Untersuchungen zu Musikpflege und Musikunterricht an den deutschen Lateinschulen vom ausgehenden Mittelalter bis um 1600, Regensburg 1969 (Kölner Beiträge zur Musikforschung, 54).

3 Vgl. zu Sturm wie überhaupt zum Schulwesen der Reformationszeit Friedrich Paulsen, Geschichte des gelehrten Unterrichts auf den deutschen Schulen und Universitäten vom Ausgang 
aber nicht nur Lateinlehrer, sondern - nach einer üblichen Konstruktion zugleich Kantor; auf dem Titelblatt seines Druckes Regenspurgischer KirchenContrapunct (Regensburg 1599; RISM R 264) heißt es: "Durch Andream Raselium M. in der newen Pfarr/ und Lateinischen Stattschul Cantorem daselbsten«.

Das Kantorenamt an der Neupfarrkirche versah Raselius äußerst gewisenhaft. Er schrieb ein Musikbuch - im Kern eine Gesangslehre - in gehobener lateinischer Diktion. Daneben komponierte und sammelte er eine groBe Anzahl von musikalischen Werken. Unter den eigenen, teils im Druck publizierten Werken, finden sich ganze Kantionalien wie auch lateinische und deutsche Motetten; in den handschriftlichen Anthologien finden sich neben eigenen Werken viele Beiträge von größeren oder kleineren Komponisten der jüngeren Vergangenheit oder Gegenwart. Eine Eigenheit der Drucke wie der Handschriften des Raselius besteht in der häufig zu findenden Modusangabe oder der modalen Anlage ganzer Sammlungen. Nach 16 Jahren in Regensburg ging Raselius wieder nach Heidelberg, um die verlockende Stelle eines Kapellmeisters am Hof Friedrichs IV. (Kurfürst von der Pfalz von 1592 bis zu seinem Tod 1610) anzutreten, der übrigens ein gebürtiger Amberger war. Er hatte sich in seiner Jugend vom Luthertum losgesagt und zum Calvinismus bekannt, was für Raselius offenbar kein Hindernis war, dem Ruf zu folgen. Er konnte die neue Stellung aber kaum mehr genießen; bereits am 6. Januar 1602 ist er, knapp vierzig Jahre alt, gestorben.

Innerhalb des regionalen Rahmens, der das Leben des Raselius umschloss, erinnert man sich auch heute noch des sonst vergessenen Schulmannes und Musikers. ${ }^{4}$ Überregional hat Raselius nicht als Person in der Fülle ihrer Aspekte Beachtung gefunden, sondern nur mit einem isolierten Teil

des Mittelalters bis zur Gegenwart. Mit besonderer Rücksicht auf den klassischen Unterricht, 3 , erw. Aufl., hrsg. von Rudolf Lehmann, 2 Bde., Leipzig 1919, das Zitat aus der Schrift De litterarum ludis recte aperiendis (1538) nach Bd. 1, S. 292.

4 Hier ist zunächst zu nennen der ehemalige Amberger Schulrektor und Raselius-Forscher Karl Schwämmlein, dem eine große Anzahl von verstreuten Einzelbeiträgen zu verdanken ist; genannt sei hier nur die zusammenfassende Arbeit: Karl Schwämmlein, Beitrag zu einer Musikgeschichte Ambergs: Andreas Raselius Ambergensis (ca. 1563 - 6. Jan. 1602), Leben und Werk, Amberg 2002 (masch.; Fotokopie u. a. in der Bischöflichen Zentralbibliothek Regensburg und in der Universitätsbibliothek Regensburg). In Regensburg bewahrt die Musikabteilung der Bischöflichen Zentralbibliothek in den Beständen der Proske-Sammlung (D-Rp) den größten und wichtigsten Teil der Kompositionen und insbesondere Anthologien des Raselius auf; ferner gibt es hier ein nach dem früheren Kantor benanntes Ensemble, den »Raselius-Chor der [evangelischen] Regensburger Kantorei« (gegründet von KMD Christian Kroll). Gunther Morche schließlich, der den Art. "Raselius, Rasel, Andreas", in: Die Musik in Geschichte und Gegenwart, 2. Aufl., hrsg. von Ludwig Finscher, Personenteil, Bd. 13, Kassel und Stuttgart 2005, Sp. 1285f., verfasst hat, war lange Jahre Universitätsmusikdirektor der Universität Heidelberg. 
seines Schaffens: einer (bis heute nicht im Faksimile verfügbaren) Schrift mit dem Titel Hexachordum seu quaestiones musicae practicae, sex capitibus comprehensae (Nürnberg 1589) sowie einer sehr umfangreichen handschriftlichen (zumeist autographen) Anthologie von Motetten (gelegentlich auch Madrigalen), die nach Modi geordnet ist und den sprechenden Titel eines Dodecachordum vivum trägt. Raselius war - wie auch andere seiner Zeitgenossen - ohne jeden Zweifel der Meinung, dass es ein universell anwendbares Ordnungsprinzip für ein- wie auch mehrstimmige Musikstücke gab: das »System «, das Glarean in seinem 1547 publizierten Dodekachordon expliziert hatte. $^{5}$

Raselius war von Glareans Ordnungsbegriffen so durchdrungen, dass er fast keine Gelegenheit versäumte, sie anzuwenden. Die Titel seiner Sammlungen (ob mit eigenen oder fremden Werken bestückt) geben ein beredtes Zeugnis. Sie lauten etwa

DODECACHORDUM | VIVUM | hoc est | DUODECIM MODORUM MU-| SICORUM EXEMPLA DUODENA | sex, quinque et quatuor vocum | in utraque scalâ admodum | insignia | Singulari judicio ac diligentia partim ex pro-| batissimorum aetatis nostrae symphonetarum mo- $\mid$ numentis recentioribus collecta, partim recens composita | per M. ANDREAM RASELIVM A. [=Ambergensem] | Gymnasii poëtici SPQ. Ratis-| ponens. Cantorem. | Mense Novembri. Anno MD XIC ${ }^{6}$

Lebendiges Dodekachordon, das ist: je zwölf herausragende Beispiele für alle zwölf musikalischen Modi, zu 6, 5 und 4 Stimmen, durchweg in beiderlei Skala [d.h. untransponiert und per $b$-molle transponiert], mit außerordentli-

5 Man könnte dabei in nachdrücklichem Sinne von einem System sprechen, insofern ein Usus von Glarean systematisch rationalisiert worden ist. Glarean kam durch konsequente Anwendung einer geringen Anzahl klar definierter Operationen (Oktavteilungen) auf einen vorgegebenen überschaubaren Bestand von Elementen (dem Tonvorrat des diatonischen Tonsystems) zu klar definierten Kategorien. sLogisch< ist seine Moduslehre weit anspruchsvoller als die choralorientierte pragmatische Lehre. Damit ist freilich nicht gesagt, dass sich das wirkliche Leben logischen Systematisierungen fügt. Desgleichen ist hier unerheblich, dass man das musiktheoretische Arbeiten mit Quinten- und Quartenspezies bis ins 11. Jahrhundert (paradigmatisch ausformuliert bei Bern von Reichenau, vgl. dazu den Beitrag von Christian Meyer im vorliegenden Band) zurückverfolgen kann. Denn Raselius und die anderen Humanisten, die sich auf Glarean beriefen, fragten nicht nach möglichen historischen Vorbildern der Lehre des Dodekachordons.

6 Titel nach dem Tenorstimmbuch; detaillierte Beschreibung der Quelle bei Gertraut Haberkamp, Die Musikhandschriften der Bischöflichen Zentralbibliothek Regensburg, Bd. 1: Sammlung Proske. Manuskripte des 16. und 17. Jahrhunderts aus den Signaturen A. R., B, C, $A N$., München 1989 (Kataloge Bayerischer Musiksammlungen, 14), S. 33-40, unter der Signatur A. R. 774. Dort sind auch viele andere Handschriften aus dem Umkreis des Raselius verzeichnet. Wichtiges Material (Faksimiles, Transkriptionen) zu dieser Quelle findet sich weiterhin in dem Band: Andreas Raselius, Cantiones Sacrae, hrsg. von Ludwig Wilhelm Roselius (Augsburg 1931), rev. Neuaufl., Wiesbaden 1972 (Denkmäler der Tonkunst in Bayern, Jg. 29/30, Bd. 36). 
chem Urteil und ebensolcher Sorgfalt, zu einem Teil auch aus den neueren Denkmälern der anerkanntesten Komponisten unserer Zeit zusammengetragen von Magister Andreas Raselius aus Amberg, dem Kantor des Gymnasium poeticum von Rat und Bevölkerung der Stadt Regensburg. Im Monat November 1589 .

oder

EXERCITATIONES | MUSICAE | Andreae Raselii A. Cantoris | apud Ratisponens. | IN QUEIS | MAGNIFICAT, (quae vocant) ad omnes DO-| DECACHORDI GLAREANI modos: | Et aliae Cantiones 654 et 8 vocum, festivitati- | bus nuptialibus amicorum diversis temporibus | compositae et ordine descriptae | Martio Mense | Anni | MDVIC [1594] | Horis subsecivis. $^{7}$

Musikalische Geistesübungen des Kantors bei den Regensburgern Andreas Raselius aus Amberg, in denen Magnificat (wie man sie nennt) nach allen Modi des Glareanischen Dodekachordon und andere Stücke zu 6, 5, 4 und 8 Stimmen, für Hochzeitsfeiern von Freunden zu verschiedenen Zeiten komponiert und in guter Ordnung niedergeschrieben. Im Monat März des Jahres 1594. Angefertigt in Mußestunden.

oder

DISCANTUS | Teutscher Sprüche/ auß den Son-| täglichen Evangeliis durchs gantze Jar/ mit sonderm | fleiß gezogen/ und mit funff stimmen gesetzt/| Durch | ANDREAM RASELIUM AMBERG. | In welchem viva exempla dodecachordi Glareani in utraque Scala | gefunden werden. Zuvor nie in Druck geben. | Allen Christlichen versammlungen Teutscher na-| tion fast dienstlich. | Gedruckt zu Nürmberg/ in der Gerlachischen Truckerey/| durch Paulum Kauffmann. | M. D. XCIII. [1594] $]^{8}$

oder

CANTUS | Teutscher Sprüche/ auff die Für-| nemsten Järlichen Fest und Aposteltäge/ auß | den gewöhnlichen Evangeliis gezogen. | Und mit 5.6.8. und 9. stimmen/ auff die | 12. modos Dodecachordi gesetzt,// Durch | ANDREAM RASELIUM AMBERG. | Gedruckt zu Nürmberg/ durch | Paulum Kauffmann. | M.D.XCV. [1595 $]^{9}$

Das sprechendste wie bekannteste Zeugnis für die Glarean-Verehrung des Raselius ist die Anthologie Dodecachordum vipum - »lebendiges Dodekachordon« oder in nüchterner logischer Formulierung: exemplarische Konkretisierungen eines zwölffältig gegliederten Potentials. Nun sind Exemplare einer bestimmten Art nur in den spezifischen Merkmalen determiniert, während andere Besonderheiten vom Allgemeinbegriff nicht erfasst werden. $\mathrm{Ob}$ nun die erfassten oder nicht erfassten Besonderheiten wesentlich oder zufäl-

7 Titel nach dem Stimmbuch der Quinta vox (erhalten nur C, B, 5); Beschreibung bei G. Haberkamp, Sammlung Proske (wie Anm. 6), S. 137-149, unter der Signatur A. R. 954.

8 RISM R 262, dort mit stark verkürztem, den Glareanbezug unterschlagendem Titelzitat.

9 RISM R 263. 
lig, essentiell oder akzidentiell sind, steht nicht a priori fest, sondern unterliegt der Bewertung. Auf Modi und Exempla bezogen heißt dies: Ein etwa dem dorischen Modus zuzurechnendes Stück stimmt in den Punkten mit dem Modus überein, die der Definition des Modus zugrunde liegen (diese Aussage ist tautologisch, aber im gegebenen Kontext vertretbar). Es lässt sich leicht zeigen (und ist im Prinzip auch allgemein bekannt), dass die Definitionsmerkmale eines Modus die Struktur eines Tonsatzes nur partiell, aber nicht in ihrer integralen Komplexität betreffen. ${ }^{10}$

Es steht außer Frage, dass Raselius die Modi und die durch sie bestimmten Merkmale für wichtig hielt. Das schließt aber in keiner Weise aus, dass es andere Merkmale an einem Musikstück gibt, die wichtig sind, aber vom Modusbegriff nicht erfasst werden. Das Modusverständnis des Raselius war zunächst bestimmt durch sein Interesse, den Sängern mittels der Erkenntnis modaler Strukturen einen von zwölf möglichen sallgemeinen Wegeverläufen anzuzeigen; wenn der Sänger gleichsam die Richtung kennt, fällt ihm das Finden der konkreten Schritte dann nicht mehr schwer. Explizite Äußerungen finden sich in der Gesangslehre des Raselius:

HEXACHORDUM | Seu | QUAESTIO- | NES MUSICAE | PRACTICAE, SEX CA- | pitibus comprehensae, quae conti- $\mid$ nent perspicua methodo ad | praxin, ut hodie est, | necessaria. | Pro Gymnasio Poëtico S. P. Q. | Ratisponensis, hoc ordine disctinctae, \& | idoneis exemplis, unà cum pulcher- | rima XII. Modorum do- | ctrina illustratae | à | M. ANDREA RASELIO, | ejusdem Cantore. | CUM GRATIA ET PRIVILEGIO | IMPERIALI. | Noribergae. | M D XIC. ${ }^{11}$

Hexachordum, oder: Fragen der praktischen Musik, gefasst in sechs Kapitel, die in deutlichen Worten alles Notwendige zum Verständnis der heutigen Praxis enthalten, geschrieben für das Gymnasium Poeticum von Senat und Volk der Stadt Regensburg, in guter Ordnung eingeteilt und mit aussagekräftigen Beispielen sowie einer ungemein schönen Lehre von den 12 Modi illustriert von Magister Andreas Raselius, dem Cantor des genannten Gymnasiums. Mit Druckerlaubnis und Kaiserlichem Privileg. Nürnberg. 1589.

10 Die Spezifikation der Diatonik in 12 (oder 8) Modi kann durchaus einzelne kompositorische Bereiche in unterscheidbarer Weise prägen, so das "Exordium«. Aber auch hier erscheinen die Auswirkungen der Moduslehre primär im Lichte der Gestaltung der Einzelstimmen, die im »Exordium « durch rhythmisch versetztes Eintreten besonders gut hörbar sind. Auch die generelle Unterscheidung zwischen authentischer und plagaler Disposition kann zu merklichen Unterschieden führen, aber auch hier wohl primär im Blick auf die Einzelstimme, nicht den Klangverlauf. Sicher spielte sadditives Komponieren zur Zeit des Kontrapunkts eine Rolle; und sicher spielte auch die Wahrnehmung des Sängers, der eben sseiner Stimme sang, eine Rolle. Aber wie groß diese Rolle war, lässt sich nicht ein für allemal feststellen.

11 Titelblatt, fol. Alr. Das Buch ist unpaginiert; zitiert wird nach dem Lagenalphabet. 
Der auf die Widmungsvorrede folgende Text ${ }^{12}$ wendet sich an den $»$ Lector amice«, insbesondere aber an die Musiklehrer und Kantoren, also die Fachgenossen des Raselius. Betont wird der auf die Schule gerichtete Zweck des Buchs und die daraus resultierende (nach der Meinung des Raselius) einfache Schreibart. Der schulische Singunterricht war kein Selbstzweck, sondern diente der Ausbildung im korrekten mehrstimmigen Singen im Hinblick auf den Gottesdienst. Das korrekte Singen aber wird gefördert, wenn man die allgemeinen Leitlinien kennt, denen die Melodien jeder Einzelstimme folgen müssen. Hilfreich ist auch die >Disposition<, die übliche >vertikale< Anordnung der Einzelstimmen. Der Weg von der gleichsam rallgemeinsten Grundlage $<$ dem von Hexachorden überzogenen diatonischen Tonsystem, hin zu den bereits weiter spezifizierten Modi des Glarean ist damit vorgezeichnet. Den Titel des Buches erklärt Raselius so, dass er in sechs Kapiteln praktisch alle Fragen abhandele, die zur Praxis »ut hodiè est « hinführen. Das Buch ist also keine Kompositionslehre, sondern eine Singlehre; der Akzent liegt nicht auf dem Erzeugen von Strukturen, sondern auf deren Erkenntnis als Mittel zur Beförderung eines fehlerfreien Vortrags.

Raselius führt zunächst die drei »claves signatae« (G-Schlüssel, CSchlüssel, F-Schlüssel) ein, um dann die drei Hexachordarten (permanens [naturalis], molle, durum) und mit ihnen zugleich die Tonfolge der Hexachorde zu bestimmen. ${ }^{13}$ Die folgenden Übungen oder Exempel ${ }^{14}$ zeigen, dass das Hexachord als skalenartige Tonfolge nur eine didaktische Reduktion ist. Es kommt in der Singlehre vielmehr darauf an, die Hexachorde (ebenso wie später die »modalen Oktaven«) gleichsam als Strukturräume zu verinnerlichen, die die konkreten Melodien vorprägen und vorhersehbar machen. Nach der Lehre der Hexachordverbindung und -mutation werden im vierten Kapitel sodann die einzelnen, prinzipiell aus den Hexachorden schon bekannten Intervalle gelehrt. Zugleich werden aber auch die Intervalle eingeführt, die den Hexachordraum übersteigen. Bei der Oktav wird dabei auf das Phänomen ihrer doppelten Teilbarkeit (»harmonicè» in Quinte plus

12 Hexachordum, fol. A5 $5^{\mathrm{v}}-\mathrm{A} 8^{\mathrm{r}}$.

13 Vgl. ebda., fol. A6 ${ }^{\mathrm{v}}-\mathrm{A} 8^{\mathrm{r}}$. Er nennt insgesamt sieben gebräuchliche Schlüsselpositionen: G2, C1, C2, C3, C4, F3, F4. Daneben spricht er von »septem distincta systematum genera« (fol. B4 ${ }^{v}$ ) gemäß der Lage des Tones $a$ im Notensystem: Das erste »systema« liegt vor bei Lage des Tones a auf der unteren Notenlinie, also bei C2-Schlüssel. C3- und C4Schlüssel bezeichnen demzufolge das dritte bzw. fünfte "genus systematis«, da die Spatien mitgezählt werden (vgl. die Beispiele auf fol. $B 5^{\mathrm{r} / v}$ ). Mit dieser Lehre stehen Angaben im Dodecachordum vivum in einem vom Verfasser nicht erklärbaren Widerspruch: Dort werden nämlich Systeme mit C4-Schlüssel dem »quartum genus«, Systeme im C3-Schlüssel gar dem »quintum genus« zugerechnet.

$14 \mathrm{Vgl}$. Hexachordum, fol. $\mathrm{B}^{\mathrm{v}}-\mathrm{C} 2^{\mathrm{r}}$. 
Quarte, »arithmeticè» in Quarte plus Quinte) hingewiesen, ${ }^{15}$ das in der Modusherleitung Glareans eine zentrale Rolle spielt:

Quid prodest haec nosse?

Ad exactam modorum musicorum cognitionem, quâ nihil in tota hac arte pulchrius aut magis musicum. Haec enim doctrina tota immediatè dependet

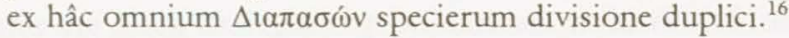

Wozu dient es uns, dies zu wissen?

Zur genauen Erkenntnis der "modi musici«, wobei es in dieser ganzen Kunst nichts gibt, das schöner oder »musikalischer« wäre. Diese ganze Lehre hängt nämlich ab von der doppelten Teilbarkeit aller Oktavspezies.

Im fünften Kapitel folgt schließlich noch ein kurzer Abriss der Mensuralnotation (Einzelnoten, Ligaturen, Sonderzeichen, tactus) ${ }^{17}$ Das umfangreiche sechste Kapitel, »De Modis musicis «, ${ }^{18}$ setzt nun den krönenden Schlussstein und behandelt ausführlich die Herleitung und Darstellung der 12 Modi (unter Ausschluss der beiden »spurii«), ihre Modifikationen hinsichtlich der Ausfüllung des Oktavambitus (»imperfecti«, "perfecti«, »excedentes«), die nochmals ausdrückliche Unterscheidung in "Authenti« und »Plagales«, die Transposition und schließlich die Stimmendisposition im mehrstimmigen Satz. Die ausführliche Besprechung jedes einzelnen Modus in transponierter und untransponierter Form wird stets durch Notenbeispiele illustriert. Hier überwiegen Kanons aus der Feder des Raselius. Ausgearbeitete motettische Sätze aber kann der Leser in den folgenden Musikdrucken finden: ${ }^{19}$ den Bußpsalmen von Alexander Utendal (RISM U 119), den Sonntagsevangelien von Homer Herpol (RISM H 5187) und der Psalmodia von Lucas Lossius (Wittenberg 1579, RISM L 2877; Erstauflage 1553). Auch Introitus, Responsorien und Antiphonen können erhellend wirken. Neben weiteren unspezifizierten Werken Utendals werden noch besonders empfohlen die (wie Raselius selbst sagt) nach acht Tonarten geordneten, mit Erlaubnis des Komponisten Orlando di Lasso gedruckten Fasciculi aliquot cantionum sacrarum (Nürnberg 1582, RISM L 937). Bei den einzelnen Modi werden dann noch weitere Beispiele genannt. ${ }^{20}$ Schließlich erklärt Raselius noch die »Modi connexi seu mixti«, worunter er die Ausdehnung von Einzelstimmen über den Verlauf des authentischen wie auch plagalen Ambitus zu einer gemeinsamen Finalis versteht (»verbunden« oder »gemischt« werden hier also bei-

15 Ebda., fol. D6" $-E 1^{r}$.

16 Ebda., fol. El ${ }^{r / v}$.

17 Ebda., fol. El ${ }^{2}-\mathrm{E}^{\mathrm{r}}$.

18 Ebda., fol. E7 $7^{\mathrm{v}}-\mathrm{L3}^{\mathrm{r}}$, das sind 88 Seiten bei einem Gesamtumfang (ohne Vorreden u. ä.) von 146 Seiten.

19 Ebda, fol. Gl:

20 Ebda., fol. $\mathrm{H}^{2}$. 
spielsweise Hypodorius und Dorius, nicht aber Dorius und Mixolydius) ${ }^{21}$

Zur Bestimmung eines Modus sind nach Raselius drei Kriterien zu beachten: erstens die Ausdehnung nach oben und unten, also der Ambitus; zweitens die Schlussnote, also die Finalis; drittens die "Phrasis«, also der modustypische Melodieverlauf. Alle drei Kriterien beziehen sich ausschließlich auf die Einzelstimme. Die beiden ersten sind trivial, man kann sie zweifelsfrei konstatieren. Interessant ist allein der Begriff der Phrasis, kommt man hier doch ohne Bewertungen, ohne die Fähigkeit nicht aus, einen speziellen, konkreten Vordergrund auf einen allgemeineren, latenten Hintergrund zu beziehen. Nur an dieser Stelle kommt ein weiteres, mit den Modi zu verbindendes Kriterium beiläufig zur Sprache: die Wahl der Kadenzorte. An den Kadenzorten als >Zwischenstationen und den mit ihnen verbundenen Schlusswendungen, die aufgrund ihrer nicht nur einzelstimmlich, sondern auch gesamtklanglich wahrnehmbaren Standardisierung »clausulae formales« heißen, läge auch das Eingangstor zu einer Betrachtung der Mehrstimmigkeit vom elementaren Ausgangspunkt des Mehrklanges her. Die Moduslehre hat als Disziplin dieses Eingangstor nicht durchschritten. Weder gereicht ihr dies zum Tadel, noch aber besagt es etwas gegen die Existenz eines Bereiches hinter dem Tor.

Raselius schreibt:

Quomodo ex Phrasi?

Phrasis ipsa cantionis melodia est, germani den Thon vocant, oder die weiß unnd melodey. Hanc ut plurimum clausulae, quas formales vocant in certis clavibus, \& commatum fine, produnt: tum semitonii observatio in $\Delta$ lak. ac $\Delta 1 \alpha \delta$. [sic] speciebus. ${ }^{22}$

Wie aus der Phrasis?

Die Phrasis ist die Melodie des Stückes selbst, die Deutschen nennen es »den Ton« oder »die Weise und Melodie«. Diese [Phrasis] geben meistens die Klauseln zu erkennen, die man »formale " nennt und die man auf gewissen Tonstufen und am Ende von Textabschnitten setzt, ebenso aber auch die Beobachtung der Halbtonschritte in den Quint- und Quartgattungen.

Deutlicher lässt sich die Fixierung des Modusbegriffs auf die Einzelstimme nicht mehr ausdrücken. Mit »Phrasis«, »Ton«, »Weise", »Melodey« verwendet Raselius vier synonyme Ausdrücke, die den konkreten Verlauf der Einzelstimme bezeichnen. Durch Analyse zu suchen sind die Klauselstufen sowie die in der Melodiebildung auffälligen Quinten- und Quartenspezies, die durch die Lage des Halbtons charakterisiert sind. Hier erscheint der Modus als Folie konkreter Melodieverläufe. Hier liegt auch der Schlüssel für ein

$21 \mathrm{Vgl}$, ebda., fol. $\mathrm{K} 3^{\mathrm{v}}-\mathrm{L} 3^{\mathrm{r}}$.

22 Ebda., fol. F8 ${ }^{v}-\mathrm{Gl}^{\mathrm{r}}$. Anders als das Wort $\Delta \mathrm{t} \alpha \alpha \alpha \omega \dot{\mathrm{v}}$ schreibt Raselius die griechischen Wörter »diapente « und »diatessaron« stets abgekürzt in der oben wiedergegebenen Form. 
Komponieren, das sich nachdrücklich und eng an Vorgaben der Glareanischen Theorie halten möchte: Je stärker die Melodien darauf angelegt sind, die jeweils modustypischen Quinten- und Quartenspezies hören zu lassen, und je enger sich die Klauseldisposition an die Vorgaben des Modus hält, desto >modaler< wird das Stück sein. ${ }^{23}$ Das Moduskapitel bei Raselius fußt ganz auf dem Gedankengut Glareans, was der Autor am Ende der Vorrede an den freundlichen Leser dankend vermerkt. Zugleich gibt er abschließend einen Hinweis auf ein Musikwerk, das er nach den 12 Modi einzurichten gedenkt. Die Auswahl ist, wie die zitierten Titel zeigen, recht groß, doch wird man in erster Linie an die Teutschen Sprïche denken, da Raselius ja von eigenen Kompositionen spricht:

In sexto capite tandem pulcherrimam certissimamque XII. Modorum doctrinam, quâ fieri potuit, perspicuitate persequi conatus sum. Quid praestiterim, tuum esto judicium, Lector benevole. Caussas [sic] ac traiones requiris? En tibi Glareani Dodecachordum. Nec addo pluria. Salve \& fave, \& probepdiem exempla 12. modorum à meâ tenui musica exspecta. Ex Musaeolo Augustae Tiberii ad Istrum. ${ }^{24}$

Im sechsten Kapitel habe ich schließlich versucht, die über alle Maßen herrliche und unverbrüchlich fest gegründete Lehre von den 12 Modi in der größtmöglichen Deutlichkeit darzustellen. Was ich hier zu leisten vermochte, bleibt deinem Urteil anheim gestellt, geneigter Leser. Verlangst Du aber Ursachen und Begründungen? Dann greife zum Dodecachordum des Glarean. Weiter füge ich nichts hinzu. Sei mir gegrüßt und bleibe mir gewogen- und erwarte in der nächsten Zeit Beispielkompositionen für die 12 Modi, die meiner bescheidenen Kompositionskunst entspringen. Aus dem Studierzimmerchen in der Römischen Kaiserstadt an der Donau.

Das Dodecachordum vivum ist das Seitenstück zum Hexachordum, enthält es doch einen reichen und wohlgeordneten Vorrat an kunstvoll ausgearbeiteten Exempelstücken zu allen Modi in untransponierter und transponierter Form. Hier interessieren nur noch zwei Eigenheiten, die auf die direkte Verbindung des Dodecachordum vivum zum wirklichen musikalischen Leben verweisen.

Im Stimmbuch »Bassus ${ }^{25}$ findet sich eine »Praefatio ad Successorem

23 Diese Art von modaler Restriktion bedeutet keineswegs, dass dieses Stück eine besonders starke ,kirchentonartliche` Färbung hätte. Die klangliche Gemeinsamkeit modaler wie snicht-modalere (nach des Raselius Meinung: miserabel komponierter) Motetten des 16. Jahrhunderts liegt im diatonischen Tonsystem begründet, nicht in Fragen der speziellen Melodiebildung, der Stimmen- oder Klauseldisposition.

24 Ebda., fol. $\mathrm{A} 7^{\mathrm{v}}-\mathrm{A} 8^{\mathrm{r}}$.

25 Die Stimmbücher sind auf dem Einband mit den gewöhnlichen Namen bezeichnet; auf den handschriftlichen Titelblättern dagegen heißt der Cantus "Vox excellens«, der Altus "Vox acuta« und der Bassus »Vox gravis«. Die übrigen drei Stimmbücher weisen keine Besonderheit auf. 
meum ${ }^{26}{ }^{26}$ Dort heißt es nach einem kurzen Abriss der für den Musikunterricht wichtigen Themen:

Quorum [sc. praecepta in Hexachordo] sexto capite, doctrinam XII modorum tot seculis obscuratam, tot excellentibus musicis (dubito num musici appellandi) ignoratam ac feré amissam, nisi Glareanus vir indefessi laboris, magni judicii, stupendae eruditionis, è tenebris eam sagacitate sua indagasset, industria reperisset, studio et diligentia eruisset, et ab interitu vindicasset, perspicue, nisi meus me animus fallat, et dextré explicavi. Quam si nosti, SUCCESSOR bone, meritò gaudeo, mihique tibique huicque scholae gratulor plurimum, ac magnae felicitatis loco pono. ${ }^{27}$

In dessen [d. h. des Buchs Hexachordum] sechstem Kapitel habe ich die Lehre der 12 Modi, wenn mich meine Meinung nicht trügt, deutlich und korrekt erklärt. Diese Lehre war so viele Jahrhunderte lang verdunkelt, sie blieb so vielen hervorragenden Musikern (ich zweifle freilich, ob sie wirklich »Musiker " genannt zu werden verdienen) unbekannt, und sie wäre fast gänzlich in Vergessenheit geraten, wenn nicht Glarean - ein Mann von unermüdlichem Fleiß, unbestechlichem Urteil, Staunen erregender Bildung - sie aus der Dunkelheit erlöst, mit seiner überragenden Geisteskraft untersucht, mit Hartnäckigkeit wiedergewonnen, mit Gelehrteneifer und Sorgfalt aus dem Staube wieder aufgerichtet und vor dem Untergang bewahrt hätte. Wenn du diese Lehre versteht, mein geschätzter Nachfolger, dann freue ich mich verdientermaßen, und ich gratuliere mir, dir und dieser Schule von ganzem Herzen, und ich achte es einem großen Glück gleich.

Die Konstruktion, dass Glarean nur eine verschüttete Wahrheit wiederentdeckt habe, dient natürlich der Legitimation seiner Lehre. Sie zeigt aber zugleich, dass ein wirklich >korrektes< Komponieren, das den aus Glareans Modi fließenden Normen gänzlich entsprochen hätte, für viele Komponisten der älteren und jüngeren Vergangenheit des Raselius gar nicht möglich war. Raselius hätte sicher starke Bedenken gegen eine undifferenzierte Verteilung von Moduszuweisungen geäußert: Die Befolgung modaler Normen adelte einen Komponisten des 16. Jahrhunderts, aber längst nicht alle gehörten zum Adel.

Im Tenor-Stimmbuch findet sich beim ersten Stück einer jeden Abteilung eine Kurzcharakteristik des betreffenden Modus nebst einigen Marginalien zum Notentext. Zur Illustration diene der Hypodorius transpositus seu

26 Zur Motivation dieser Vorrede sagt Raselius, dass er sein weiteres Schicksal nicht vorhersehen könne - sei es, dass ihn der Tod ereile, sei es, dass er eine andere Stellung annehmen sollte. Die imposante Sammlung aber sollte auch nach seinem Weggang in Regensburg verbleiben - wie es denn bis heute der Fall ist. Vgl. den Abdruck der Vorrede in: Andreas Raselius, Cantiones sacrae (wie Anm. 6), S. XLVIf. (mit Druckfehler "ad Successorum«).

27 Ebda., S. XLVI. 

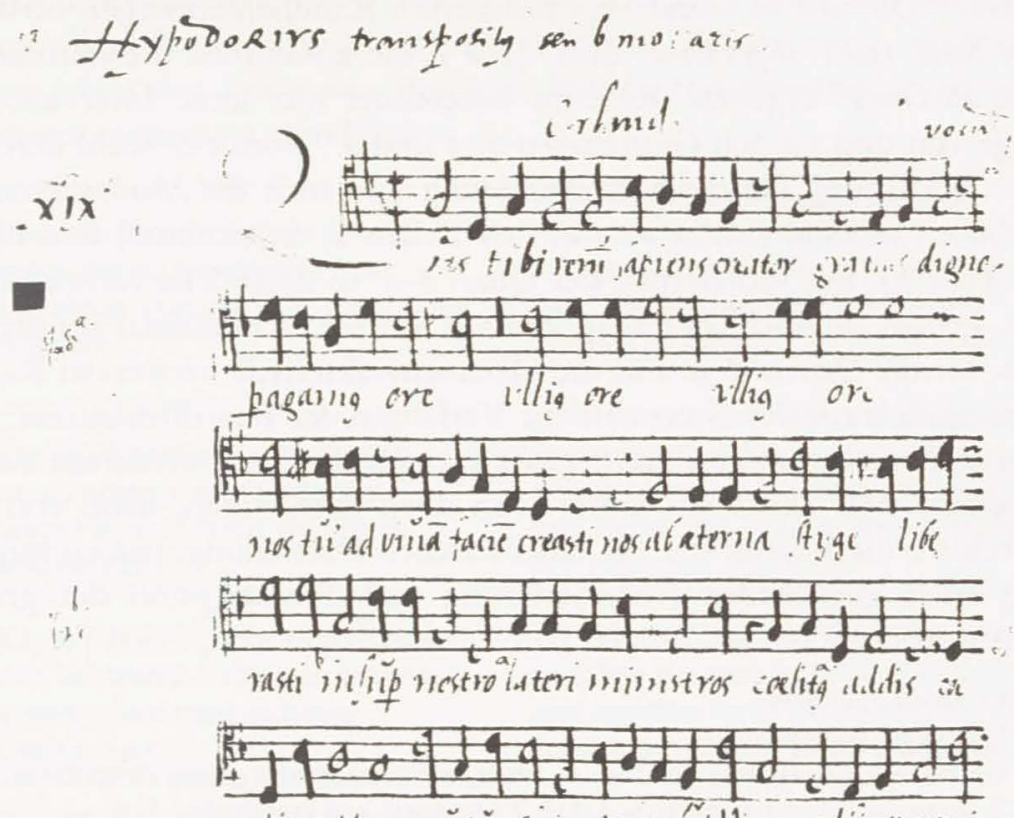

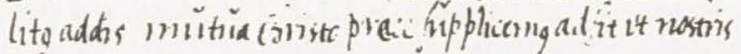
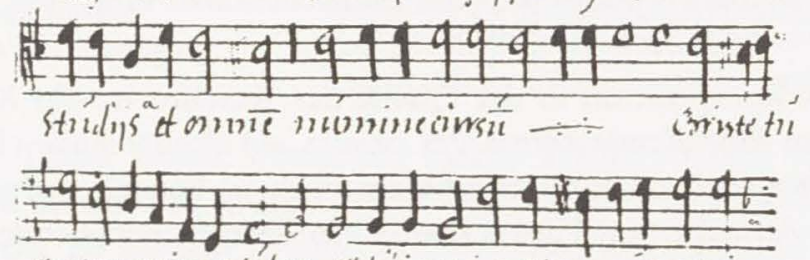

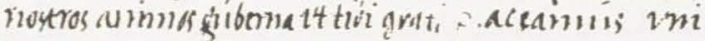

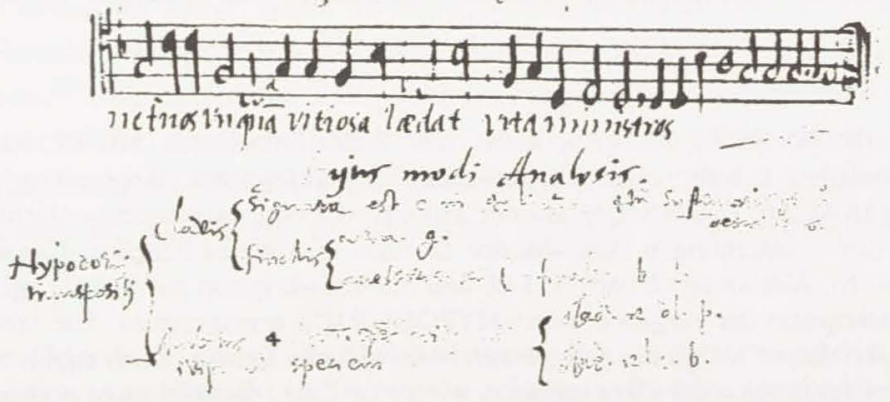

Abbildung 1: Andreas Raselius, Dodecachordum vivum, Stimmbuch Tenor, Beginn des 19. Stücks (Orlando di Lasso, Quas tibi rerum, LV 721, Kontrafaktur) mit Anmerkungen von Raselius zur Struktur des Modus (D-Rp, A. R. 774) 
$b$ mollaris. ${ }^{28}$ Die mit »a. " und »b. indizierten Randbemerkungen verweisen auf die $\Delta \downarrow \alpha \delta$. (hier: $d-g)$ bzw. $\Delta 1 \alpha \varepsilon$. $\left(g-d^{\prime}\right)$, die zusammen die arithmetisch geteilte D-Oktav ergeben. Raselius bezeichnet nur leere Intervalle, also

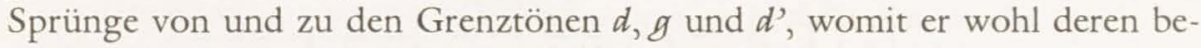
sondere Bedeutung für die Melodiebildung wie auch die Moduserkenntnis hervorheben möchte (Index "a." in den Zeilen 1, 4 [zweimal] und 8, "b." nur in Zeile 4). Die Indizierung der Quart $a-d^{\prime}$ in Zeile 6 ist vermutlich ein Irrtum, gehört sie doch im transponierten System nicht einmal zur gleichen Spezies wie die Quarte $d-g$. Für die Moduscharakteristik verwendet Raselius hier wie auch sonst das übersichtliche Verfahren der Begriffsdihairese: jeder Oberbegriff wird in zwei Unterbegriffe zerlegt, die dann wiederum zweigeteilt werden. Die Umschrift lautet (von oben nach unten, dann von links nach rechts ohne Bewahrung der tabellarischen Darstellung, jedoch mit Einfügung einer gliedernden Nummerierung und Transkription der griechischen Ausdrücke):

Hujus modi Analysis.

1. Hypodorij transpositj -

1.I Clavis - 1.1.1 signata est $\mathrm{c}$ in .4. linea, $4^{\mathrm{rum}}$ systematis genus describens. -

1.1.2 finalis - 1.1.2.1 vera. g. - 1.1.2.2 accesoria seû confinales. b.d. -

1.2 Ambitus 4 diapason species arithmeticè divisâ in speciem - 1.2.1 diatessaron re sol p. ${ }^{29}-1.2 .2$ diapente re la. b.

Lassos Komposition in der Quelle des Raselius ist ein Kuriosum. Dass es sich um eine Kontrafaktur des Stücks »O decus celsi« (LV 721; der Text besteht aus vier sapphischen Odenstrophen) handelt, ist im Supplement der neuen Ausgabe von Lassos sämtlichen Werken verzeichnet. ${ }^{30}$ Diese Motette nimmt in der Umgebung ihres Erstdruckes eine von der Lasso-Forschung

28 Vgl. die Abbildung 1 (D-Rp, A. R. 774, Tenor-Stimmbuch, Nr. 19; Reproduktion mit freundlicher Erlaubnis der Bischöflichen Zentralbibliothek, Regensburg). Vgl. auch die Abbildung der Tenorstimme aus der Motette "In voce exultationis« (Jacob Handl/Gallus) mit dem Anmerkungen zum "Modus Dorius« in Andreas Raselius, Cantiones sacrae (wie Anm. 6), Abbildung. 2 nach S. LIX und die Transkription auf S. LII; vgl. ebda. auch die Transkription der Angaben zum »HYPODORIUS transpositus«. Die von Raselius nicht transkribierten Marginalien verweisen beim »Modus Dorius« noch eigens auf geringfügige Überschreitungen des Oktavambitus, wie sie im Lasso-Beispiel nicht vorkommen.

29 »p.«steht nach Ausweis anderer Einträge für »(hexachordi) permanentis«, womit in der Nomenklatur des Raselius das Hexachordum naturale gemeint ist.

30 Orlando di Lasso, Sämtliche Werke, Supplement: Horst Leuchtmann und Bernhold Schmid, Seine Werke in zeitgenössischen Drucken 1555-1687, Bd. 3: Register, Kassel 2001,

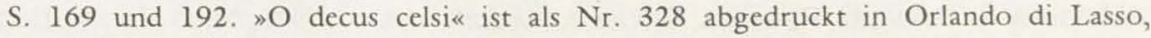
Sämmtliche Werke, Bd. 11: Magnum opus musicum. Lateinische Gesänge für 2, 3, 4, 5, 6, 7, 8, 9, 10 u. 12 Stimmen, Tl. VI: Für 5 und 6 Stimmen, hrsg. von Carl Proske und Franz Xaver Haberl, Leipzig 1900, S. $156 f$. 
mehrfach behandelte Sonderstellung ein. ${ }^{31}$ Der im Dodecachordum vivum unterlegte Text lautet (die in der abgebildeten Tenorstimme aufgrund von Pausen fehlenden Verse wurden nach dem Cantus-Stimmbuch ergänzt; die Übersetzung stammt vom Verf.):

Quas tibi rerum sapiens creator gratias digno peragamus ore Heu situm non est id in eloquentis ullius ore.

Nos tu ad vivam faciem creasti nos $a b$ aeterna styge liberasti insuper nostro lateri ministros caelitus addis.

Mutua Christo prece supplicemus adsit ut nostris studiis et omnem dextero vitae tueatur huius numine cursum.

Christe tu nostros animos guberna ut tibi grati placeamus uni ne tuos unquam vitiosa laedat vita ministros.
Welchen Dank könnten wir dir, weiser Schöpfer der Dinge, mit geziemenden Worten abstatten? Ach, das liegt in keines beredten Menschen Mund!

Du hast uns nach deinem lebendigen Antlitz geschaffen, von der ewigen Verdammnis erlöst, und stellst uns dazu noch die Helfer des Himmels zur Seite.

Im Wechsel lasst uns Christus anflehen, er möge unseren Studien nahe sein und unseren ferneren Lebenslauf mit seinem göttlichen Walten beschützen.

Christus, leite du unsere Seelen, damit wir dir allein angenehm gefallen, auf dass niemals ein liederliches Leben deinen Dienern schaden möge.

Die Herkunft des Textes konnte wenigstens partiell geklärt werden. Drei der vier sapphischen Strophen sind einem Gedicht von Johann Stigel (1515-1562) mit der Überschrift De Angelis hymnus entnommen, das posthum in der Sammlung Poematum Liber (Jena 1566) gedruckt wurde; die Strophen 1, 2 und 4 der Motette entsprechen den drei Schlussstrophen 20-22 des langen Engelshymnus. ${ }^{32}$ Die dritte Strophe war bislang nicht nachzuweisen; es ist aufgrund ihrer Nähe zur Schulsituation immerhin denkbar, dass sie von Raselius selbst stammt. Stigel passt gut in die lokale und geistige Umgebung des Raselius: „Und als er treffliche Proben seiner Poetischen Ge-

31 Vgl. zuletzt Orlando di Lasso, The Complete Motets, Bd. 13: Mottetta, sex vocum, typis nondum uspiam excusa (Munich, 1582), hrsg. von Rebecca Wagner Oettinger, Middleton 2005, Introduction, S. XV (Text »O decus celsi« - Herkunftsangabe: »from a play on the life of Esther « - mit englischer Übersetzung, S. XXIII; Partitur, S. 43-47).

32 Deshalb wäre der Text wohl kaum je nachzuweisen gewesen ohne die Hilfe eines von der DFG geförderten profunden wissenschaftlichen Projektes der Universitäten Heidelberg und Mannheim zur neulateinischen Dichtung: CAMENA - Lateinische Texte der Frühen Neuzeit. Corpus Automatum Multiplex Electorum Neolatinitatis Auctorum (http://www.unimannheim.de/mateo/camena; dort auch nähere Angaben zu den Betreibern). Der Hymnus steht im Poematum Ioannis Stigelii Liber I. Continens Sacra auf fol. $\mathrm{K}^{\mathrm{v}}$ - $\mathrm{L} 1^{\mathrm{r}}$. 
schicklichkeit abgeleget hatte: so ward er vom Kayser Carl V. zu Regenspurg zum Poeten gecrönet ... Uebrigens war er D. Luthers und D. Melanchthonis [sic] vertrautester Freund. $\aleph^{33}$

Doch zurück zu Raselius' Anmerkungen. Die modale Analyse ist eine Analyse des Melodieverlaufs, nicht nur des Tenors, sondern prinzipiell einer jeden Einzelstimme. Überspitzt gesagt: das mehrstimmige Stück gehört zum transponierten hypodorischen Modus in einem klar zu umreißenden Sinn nur dann, wenn man nicht das Ganze, sondern die Summe seiner Teile ins Auge fasst. Man schlage im übrigen die Partitur des Stücks nach; ohne Ansprechen des >vertikal-akkordischen< Moments dürfte gerade dieser Satz nicht angemessen zu beschreiben sein. Die Moduslehre liefert dafür keine Handhabe; sie will etwas anderes, verbietet andererseits aber auch nicht den Blick auf das, womit sie sich nicht explizit beschäftigt.

Insbesondere das Hexachordum des Raselius wurde - neben vielen anderen Schriften - von Bernhard Meier ${ }^{34}$ immer wieder zum Zeugen nicht dafür aufgerufen, dass es »Modi« gab. Ein Problem entsteht aber aus der impliziten Behauptung, dass diese »Modi den Status von »Tonarten der klassischen Vokalpolyphonie" gehabt hätten, die modernem harmonischem Denken auf gleicher theoretischer Höhe und mit gleichem Bezugsfeld diametral entgegengesetzt gewesen wären, so dass die Anwendung der modernen Kategorien auf das alte Repertoire notwendig zu verzerrten und falschen Aussagen führen würde. Strittig ist nicht, dass man vokalpolyphone Stücke modal (ob in 8 oder 12 Modi, spielt keine entscheidende Rolle) ordnen kann. Strittig sein kann nur die wissenschaftstheorisch wie auch wahrnehmungspsychologisch komplexe Frage, ob denn die Konzepte der Modi einerseits, der harmonischen Tonalität andererseits überhaupt auf ein und derselben Ebene angesiedelt sind, ob sie also in vergleichbarer Weise beanspruchen, die klangliche Kohärenz von Musikstücken umfassend zu begründen. Meiers Buch ist als Abhandlung über modale Konzeptionen und Urteilsweisen in Explikationen von Autoren des 16. Jahrhunderts ungemein hilfreich, blendet aber das eigentliche Problem: in welchem Sinne »Modi« als »Tonarten« über ihre bloße Klassifikationsfunktion hinaus zu betrachten sind, dadurch aus, dass von vornherein durch die äquivoke Verwendung des Ausdrucks »Ton-

33 Art. "Stigelius oder Stiegel, (Johann)«, in: Johann Heinrich Zedler, Großes vollständiges Universallexikon aller Wissenschaften und Künste, Bd. 40, Halle 1744, Reprint Graz 1962 und 1997, Sp. 80. Die Krönung zum Poeta laureatus fand beim Reichstag 1541 statt; während dieses Aufenthalts soll Stigel nach einem Bericht Melanchthons eine Regensburger Bürgersfrau gerettet haben, die von einem Spanier bedrängt wurde (vgl. ebda., Sp. 81).

34 Bernhard Meier, Die Tonarten der Klassischen Vokalpolyphonie. Nach den Quellen dargestellt, Utrecht 1974 (vgl. die zahlreichen Einträge unter "Raselius« im Personenverzeichnis, S. 470$)$. 
arten « eine kategoriale Identität postuliert wird ${ }^{35}$ Dadurch geraten »Modi«, die doch letztlich immer von der Einstimmigkeit her argumentieren, und »harmonische Tonalität«, deren entwickelte Theorien von der »Harmonie» als stationärem wie auch in die zeitliche Sukzession entfalteten Relationsphänomen ausgehen, in einen künstlichen Gegensatz. Man hat sich nicht für oder gegen das eine oder andere zu entscheiden, sondern anzuerkennen, dass sich Mehrstimmigkeit grundsätzlich zwischen den Polen des >Stimmlichen< und des > Klanglichen $<$ bewegt. Welche Pole eine Theorie akzentuiert, ist nicht durch Verweis auf vermeintliche ‘Quellen festzulegen, sondern beruht auf einer Entscheidung, die vom Erkenntnisinteresse wie auch vom Urteil über das historisch Angemessene und systematisch Vertretbare abhängt. Autoren wie Glarean (oder Raselius) kann oder soll man zu Rate ziehen; sie nehmen einem die Entscheidung aber nicht $a b .^{36}$

Die Begeisterung des Raselius für die Person und die Leistung des Glarean mag man in erster Instanz mit der unbestreitbar nützlichen und wichtigen Ordnungsfunktion erklären, die diese Lehre im Umgang mit den scheinbar so uniformen polyphonen Kompositionen um 1600 ausüben konnte. Wollte man sich aber allein auf diese Begründung beschränken, griffe man wohl zu kurz. Deshalb sei noch versucht, die Affinität von Raselius zu Glarean auch mit dem Faktum ihrer gemeinsamen Verwurzelung in der lateinisch verfassten, zudem griechisch inspirierten Geisteswelt der $\mathrm{Hu}$ manisten in Verbindung zu bringen. Wenn wir ein Buch wie das Hexachordum des Raselius (vom Dodekachordon des Glarean zu schweigen) nur im Hinblick auf seinen vermeintlichen >Sachgehalt< lesen oder diesen >Sachgehalt< allein in den musikalisch-technischen Bestimmungen orten, ${ }^{37}$ werden

35 Bezeichnend für diese Tendenz ist etwa ein Aufsatztitel wie der folgende: Bernhard Meier, "Auf der Grenze von modalem und dur-moll-tonalem System «, in: Basler Jabrbuch für bistorische Musikpraxis 16 (1992), S. 53-69.

36 Die Polemik, die sich an Meiers Buch anschloss, sollte heute nur noch als methodischer Impuls verstanden werden. Arbeiten des Typs Wer hat wann welche Meinung über die Modi vertreten? « sollten stets ergänzt werden um die Frage danach, was wir denn über ein Stück Musik wissen, wenn wir seinen Modus kennen. Noch immer lohnt die Lektüre des Beitrags von Harold S. Powers, "Tonal Types and Modal Categories in Renaissance Polyphony«, in: Journal of the American Musicological Society 34 (1981), S. 428-470, der ohne Antworten auf Fragen zu geben, die man sim Allgemeinen< vielleicht gar nicht beantworten kann - den Punkt bezeichnet, an dem das Referat von Modustheorien (aber auch von Begriffen wie »tonal types«) in die kritische Befragung ihres Erkenntniswertes übergehen muss.

37 Solche Überlegungen zur Bedeutung der Sprache erklären vielleicht die Schärfe der Rezension, die der promovierte Altphilologe Philipp Spitta über die Glarean-Übersetzung Emil Bohns verfasste, die doch über die stechnische Seiter der Glareanischen Theorie hinlänglichen Aufschluss vermittelt; vgl. Philipp Spitta, "GLAREANI Dodecachordon. Basileae MDXLVII. Übersetzt und übertragen von Peter Bohn. XVI. und XVII. Jahrgang der 
wir das Latein und eine zuweilen umständlich wirkende Terminologie als lästiges Beiwerk betrachten. Der technische >Sachgehalt< aber kann auf ein zuweilen erstaunlich geringes Maß schrumpfen. Wer sich in der Moduslehre auskennt, bedarf wohl nur der mittlerweile wieder geläufigen Stichworte, um sich ein Bild vom Inhalt des Schlusskapitels des Hexachordum von Raselius machen zu können.

Bleibt man sich aber des Phänomens bewusst, dass die Latinität als Repräsentantin der säkularen wie theologischen Geisteswelt das Wirken und Selbstverständnis von humanistisch gebildeten Musikern und Kantoren trug, dann hat Glarean die Musik in demjenigen sprachlichen Medium behandelt, das sie als Teil des großen, überzeitlich gültigen und damit auch aktuellen geistigen Universums ausweist, in dem sich gerade die Humanisten beheimatet fühlten. Der kunstvolle lateinische Ausdruck ist kein bloßes Akzidens des humanistischen Musikschrifttums, sondern Ausdruck der Überzeugung, dass Erkenntnis der Sprache bedürfe; »res et verba« bilden eine untrennbare Einheit.

Wie fern uns heute die in der Latinität der Humanisten vorausgesetzte Geisteswelt geworden und wie gefährlich die Suggestion eines gleichsam unmittelbaren und unverstellten Zugangs zum vermeintlichen Kern älteren Musikdenkens ist, mag der Blick auf das Ende der Vorrede des Hexachordum zeigen. Der Autor, der sich nochmals an den Regensburger Magistrat als seinen Dienstherrn mit der Bitte um Schutz vor Neidern wendet, bemüht den Topos der prophylaktischen Verteidigung gegen (damals wie heute) drohende Schmähkritik. Er schreibt:

Quam periculosum sit hoc seculo, Momorum \& Samiorum Lucumonum (qui, quod laus est, vitium faciunt) feracißimo, huiusmodi quippiam scriptis prodere, praeclarè scio. Neque ambigo, quin multi Aetolicam hanc audaciam sint clamaturi: \& quasi hoc nesciverint, priusquam Theognis nasceretur? Ego vero si pueritiae hoc meo labore inserviam, probemque doctis, quique de his studiis dexterius quàm vulgus judicant, de aliis sanè manum non verterim. Quis enim ille Thales, qui hoc statu rerum neminem offendat.

Ich weiß es nur allzu gut, wie gefährlich es in diesem unseren Jahrhundert ist, das äußerst fruchtbar ist an ${ }^{*}$ Momi und ${ }^{*}$ Samii Lucumones (die das, was eigentlich ein lobenswerter Vorzug ist, als einen Fehler tadeln), irgendetwas dieser Art in Schriftform herauszubringen. Und ich zweifle auch nicht, dass es viele geben wird, die dies als eine *aetolische Kühnheit brandmarken werden, und die gleichsam dies alles schon gewusst haben, bevor ${ }^{*}$ Theognis geboren wurde. Wenn ich aber der Schuljugend mit dieser meiner Arbeit einen Dienst erweisen und bei den Gelehrten sowie all denjenigen, die über derartige Studien ein ausgewogeneres Urteil haben, als das gewöhnliche Volk, Bei- 
fall erwerben kann, dann werde ich freilich von anderen Arbeiten gewiss die Hand nicht abwenden. Denn wo wäre jener * Thales, der in der gegenwärtigen Sachlage niemandem auf die Füße träte?

Die Übersetzung des Wortlauts bedeutet hier erkennbar noch nicht das Verständnis des Gemeinten; die erklärungsbedürftigen Stellen wurden in der Übersetzung durch einen Asteriskus markiert. Allenfalls Momus als Personifikation eines Gutachters oder notorischen Nörglers ist bekannt, den Musikfreunden wenigstens aus dem Text zu Bachs weltlicher Kantate Der Streit zwischen Phöbus und Pan (BWV 201). ${ }^{38}$ Die Wendung "Samius Lucumo« aber geht aus von der Bezeichnung »Lucumo« als Namen für Magnaten und Priester bei den Etruskern; mit der Verbindung "Samius Lucumo« wird sodann speziell die Figur des Pythagoras aus Samos bezeichnet. Von hier aus verallgemeinert sich über Ausonius (Epist. 4,70) ${ }^{39}$ die Wendung $» S$ amius lucumo« in Verbindung mit der Eigenschaft des »acumen« (des Scharfsinns) hin zu einer - allerdings nicht slandläufigen, deshalb für gelehrte Humanisten gut verwendbaren - Bezeichnung für einen sich ungemein klug dünkenden Menschen (wohl mit pejorativem Beiklang). Die "aetolische Kühnheit « dürfte sich nicht allein auf das dem Volk der Aetoler zugeschriebene rohe Ungestüm berufen, sondern speziell auf den als maetolius heros « apostrophierten Diomedes, der sich im Trojanischen Krieg unsterblich gemacht hatte. Theognis von Megara galt als ein Dichter, der einen Spruch für jede Lebenslage bereithielt. Unter Berufung auf Aulus Gellius (Noctes Atticae 13, 19; die Stelle beruft sich ihrerseits auf den Satiriker Lucilius) meint man mit einem Wissen, das man auch ohne Theognis (also »vor seiner Geburt«) schon hatte, eine Ansammlung von >Binsenweisheiten< und trivialen Selbst-

38 Die folgenden Erläuterungen basieren größtenteils auf Hinweisen meines Kollegen Georg Rechenauer (Lehrstuhl für Klassische Philologie der Universität Regensburg), dem ich für seine freundliche Hilfe danke.

39 Ausonius war in der Bibliothek des Raselius vertreten, vgl. Karl Schwämmlein, »Die Bibliothek des Andreas Raselius Ambergensis", in: Der Eisengau. Eine Sammlung heimatkundlicher Beiträge aus der Stadt Amberg und dem Landkreis Amberg-Sulzbach, Bd. 1, Amberg 1992, S. 53-77; der Hinweis auf Ausonius (ohne Spezifikation des Werks) auf S. 65. Die fast 600 Bände umfassende Bibliothek des Raselius ist dokumentiert in einem kurz nach seinem Tod angefertigten handschriftlichen Index Librorum Bibliothecae Raselianae. Die weitaus meisten Bücher sind in lateinischer Sprache verfasst; aber es gibt auch griechische und deutsche Schriften. Der Bildungshorizont des Humanisten Raselius war immens: Homer, Aristoteles, die lateinischen Klassiker, teils in kommentierten Ausgaben, aber auch die neuesten theologischen Abhandlungen standen einträchtig nebeneinander. Die Reproduktion der ersten Seite der "Libri Musici« im genannten Aufsatz (S. 63) zeigt "Glareani Dodecachordon « an erster Stelle; auch die von Johannes Wonnegger herausgegebene $\mathrm{Mu}$ sicae epitome ... ex Glareani Dodecachordo (Basel 1557) befand sich in der zweiten Auflage (Basel 1559) in Raselius' Besitz (vgl. ebda., S. 69). 
verständlichkeiten. ${ }^{40}$ Thales schließlich ist in der antiken Literatur zur Figur des allseits kundigen und fähigen Mannes, zur Gestalt des Tausendsassas geworden (z. B. bei Aristophanes, Vögel, 995f.). All diese Bezüge müssen heute mühsam vergegenwärtigt werden. Für Raselius und sein gebildetes Umfeld waren sie Teil ihrer Welt, ihrer »Kultur «.

Mag der an klanglich-harmonisch fundierten Kohärenztheorien der mehrstimmigen Musik interessierte moderne Leser bei den humanistischen Modustheoretikern nur wenig Anregung finden, so darf doch aus falschen Erwartungen kein Verdikt folgen. Autoren wie Glarean operierten in einem anderen Koordinatensystem: Glarean war es gelungen, auf einem eleganten Weg der Herleitung in der Verbindung von alten und neuen Momenten auf einem hohen sprachlich-intellektuellen Niveau eine Klassifikationsmethode für mehrstimmige Kompositionen zu formulieren, die neben oder nach der Klassifikation auch die Interpretation erlaubte. Musikstücke konnten den Werken der klassischen Literatur ebenbürtig an die Seite gestellt werden, weil ihre innere und äußere Ordnung gesichert war. Die Musik wurde aus der Unverbindlichkeit des willkürlich Geschaffenen und nur die Sinne Berührenden befreit. Glareans Dodekachordon erlaubte es, Musik als Gegenstand zugleich regulierter wie ingeniöser menschlicher Kunstübung zur (lateinischen) Sprache zu bringen. Nicht nur auf der Seite der »res«, sondern eng verbunden damit auch auf der Seite der »verba« mag so die Bewunderung humanistischer Kantoren vom Schlage des Andreas Raselius für Glarean und seine "pulcherrima XII. Modorum doctrina« verständlich werden.

40 Ich halte deshalb im schwer durchschaubaren Gefüge der mehrfachen Verneinungen nur die oben wiedergegebene Übersetzung von »nesciverint« für plausibel. 